\title{
Adopting Knowledge Technology to "Manage" Care: Issues and Status of Physician Use
}

\begin{abstract}
OBJECTIVE: To determine the extent to which primary care physicians have been willing to incorporate computerized protocols and clinical information systems into their practices.
\end{abstract}

DESIGN: A survey was fielded to both primary care and nonhospital-based specialty group practices of five physicians or more.

MAIN OUTCOME MEASURES: The types of these technologies in use and the degree to which the physicians themselves were active users.

RESULTS: Findings $(n=197$ or a $38 \%$ response rate) are presented grouped by three stages of software application adoption: (1) transaction; (2) decision support; and (3) expert systems or simulation of human thought. Transaction systems, particularly applications that support practice administration such as'registration, billing, and scheduling, have the highest percentage of adoption $(82 \%$ to $97 \%)$. Expert systems are uncommon $(3 \%$ to $6 \%)$, with slightly higher penetration of telephone triage (such as "ask-a-nurse") and prevention reminders applications (11\% and $19 \%$, respectively). Within group practices, physician "hands-on" use of systems is low for viewing $(\mathbf{2 8 \%})$, and even lower for entering patient information $(6 \%$ to $8 \%$ )

CONCLUSIONS: Emerging knowledge technologies such as medical decision support or expert systems are not widely accepted and may even threat-

Aulhors

FERN FITZHENRY, R.N., Ph.D. is Infomation Services Consultant with Vanderbilt University Medical Center, Nashvillc, TN; ]. WARREN SALMON, Ph. D., is Professor, University of Illinois at Chicago College of Pharmacy, Chicago, IL; PAUL A. REICHELT, Ph.D., is Associate Professor, University of Illinois at Chicago College of Nursing.

AUTHOR CORRESPONDENCE: Fern FizHenry, R.N., Ph.D., Vanderbil University Medical Center, Information Management, VUH, B-13I, 1161 Twenty-first Ave South, Nashville, TN 37232.

Copyright $\odot 2000$ Academy of Managed Care Pharmacy, Inc. All rights reserved. en traditional physician domains of expertise. Explicit consideration of potential physician opposition should be included in planning pharmaceutical managementstrategies that depend on "hands-on" physician computer use to be successful.

KEYWORDS: Knowledge, Adoption Protocols, Expert systems, Physicians, Benefit management, Managed care

J Managed Care Pharm 2000: 35-41

\author{
by Fern FitzHenry, J. Warren Salmon, and \\ Paul A. Reichelt
}

$\mathrm{T}$ he development of practice guidelines, protocols, and clinical information systems has widely outpaced the use of these tools by physicians. The unwillingness of some physicians to adopt these "soft" technologies limits their widespread utility (not only for physicians, but also for pharmacists, nurses, and allied health professionals) in making needed improvements in the quality of health care and increasing efficiency.

\section{Is Physician Use Required?}

Information technology can provide a path to improved realtime, information-driven clinical care. ${ }^{1.3}$ But when managed care organizations (MCOs) consider using clinical information systems to improve care and reduce costs by providing decision support to physicians on "best practices" in pharmacotherapy and disease management, they must consider the very limited degree to which the medical profession has adopted these systems.

The single most important force determining the cost and quality of health care today remains the physician. Eisenberg estimates that physicians directly control the spending of $90 \%$ of health care dollars." Each physician's decisions account for an estimated annual average of $\$ 500,000$ to $\$ 800,000$ of health care expenditures, not including their own fees. ${ }^{5}$ Changing physician behavior is thus crucial to reducing health care costs or modifying delivery systems. ${ }^{6}$

Many physicians would agree that an information system that organizes health care reference information and makes it easily accessible to them would be acceptable or even desirable. More reference literature is published in health care today than the typical practicing physician can follow, review, and adopt. ${ }^{7.8}$ So much information is being generated, Gross and colleagues believe, that physicians actually attend to less of it, arbitrarily reducing information to a manageable level.?

If the physician never looks at disease management protocols or enters prescriptions and orders into a clinical information system, then that system cannor provide interactive feedback to the person who can take action. Systems that provide information to physician's office personnel will not change patient care, because only the physician is qualified to alter the treatment plan. At best, feedback on ways to improve quality or lower costs will only be retrospective. 
In pharmacy, most work has become semiautomated. ${ }^{9}$ In contrast, the core work of physicians-diagnosis and treatment, as well as creating the patient medical record-is still essentially manual. ${ }^{10-12}$ Most physicians do not actively use information technology for their core work. The critical issues that affect physician use of information systems are not technical, but social. . $^{10,13-14}$ Information technology allows third parties to monitor physician performance and place restrictions on diagnosis and treatment decisions. Technology can also reduce demand for physician services by creating units of knowledge that can be delegated to nurses, clinical pharmacists, or other nonphysicians. ${ }^{15.17}$

Physicians have, to some degree, the power to veto information technology systems in use for patient care. ${ }^{18-19}$ The power of physicians to restrain the use of computers in the critical processes of patient care is unequalled in any other health care profession. Information systems accepted by nonphysicians--including those that integrate medical and pharmacy data-might not be implemented simply because physicians reject the system. ${ }^{12,19-21}$ Ultimately, physicians must at least condone the use of clinical information systems for pharmacy benefit or disease management if such systems are to be successfully implemented.

An early and prominent example of how physician opposition can halt a clinical information system occurred in 1977 at the University of Vermont. Dr. Laurence Weed computerized his manual Problem Oriented Medical Record to create the Problem Oriented Medical Record Information System (PROMIS). PROMIS mandated that physicians follow "expert rules" of data collection before making diagnosis and treatment decisions. The Department of Medicine at the University of Vermont endorsed codeveloping PROMIS over a four-year period. During the pilot, some physicians and most nurses responded favorably, but many physician users entered "free text" rather than selecting formatted responses, enabling them to escape the expert rules. Although pharmacists-long-time proponents of computerized systemswere strong advocates of the system, the physician staff voted to discontinue use of PROMIS, and the entire system was scuttled despite the enormous labor and capital investment. ${ }^{21.22}$

\section{System Classification Framework}

Historically, computer technology has been adopted in stages that also define levels of organizational impact. ${ }^{23,24}$ Early in the history of computers, Leavitt and Whisler defined three stages of information systems that are still valid today: (1) creation of large-scale data handling or transaction systems; (2) development of decision support systems applying statistical techniques to databases; and (3) simulation of human thinking with computers. ${ }^{23}$ By the early 1990s, most industries in the U.S. had two decades of experience in data handling and had advanced into using technology to improve efficiency and decision making. Many businesses were experimenting with the use of technology to simulate human thinking. The health care sector has lagged behind.

Although the first stage of information system use-large-scale data handling or transaction systems-is common in the health care industry, the second and third stages are not. A 1997 study of 6,201 hospitals revealed that 5,423 had financial systems. ${ }^{25}$
Another study indicated $75 \%$ of clinics had financial systems. ${ }^{26}$ However, information systems to support physician decision making or simulate human thinking have not been widely adopted in health care, although such applications have been developed and are available. ${ }^{27-29}$ In 1992, an industry expert estimated that no more than ten clinical decision support systems were in active use. ${ }^{30}$

Typically, potential users resist the advent of information technology because they see the use of these systems as a threat to themselves and their autonomy. These threats parallel the categories in the framework described above. Staff and clerical users of large-scale data handling systems are threatened by monitoring and control at the task level. In the next two stages, greater surveillance of resource utilization decisions threatens professional and managerial users. At the top level, executives who use information systems less intensively face the threat posed by having to rationalize previously unstructured decisions.

\section{- Physician Opposition To Information Technology}

Three studies have reported direct measures of physician opposition to medical decision support. Teach and Shortliffe surveyed physicians attending a seminar on artificial intelligence in medicine, as well as a group of local physicians. Physicians favored automation for core physician work such as diagnosis and treatment only when applications were presented as clinical aids, such as Medline, rather than replacements, such as computer-aided diagnosis. ${ }^{31}$ Another study of academic physicians, private practitioners, and house staff revealed that almost $70 \%$ of the physicians thought the computer was desirable as a source of summaries of published research, but only $28 \%$ wanted a computer to provide probability estimates for a diagnosis, even using patient clinical data as the basis for the probabilities. ${ }^{32} \mathrm{~A}$ third survey that measured attitudes of physicians, residents, and medical students at a large academic hospital found that physicians viewed large-scale data management systems, such as charge capture and pharmacy transaction handling systems, as more desirable than medical decision support systems. They opposed systems that would permit allied health professionals to take on even a limited number of traditional physician activities. While they believed computers would lower costs and improve quality, they also believed computers would result in a loss of physician autonomy. ${ }^{18}$

Physician opposition to computerizing medical decision making has been expressed more subtly than the resistance of blue collar and office workers to information technology. ${ }^{33-35}$ Physicians' refusal to adopt a computer system is commonly ascribed to technical barriers, such as a lack of features or training, rather than to their opposition to using any and all computer information systems. This perception has caused much research in medical informatics to focus on technical barriers to physician acceptance of clinical information systems. ${ }^{30.36-37}$

Significant technical barriers to physician acceptance do, in fact, exist. Physician data entry in an automated patient record disrupts current procedures and takes more time than entry in a manual patient record. ${ }^{38}$ Seamless incorporation of physician 
use is impossible. Even automated flowsheets impose data entry requirements not required by the manual record. Unfortunately, clerical input of physician data is expensive. ${ }^{39}$ In 1998, for a major academic medical center in the central states, the annual cost of physician transcription for even a select subset of dictated notes was estimated at $\$ 325,000$.

Information system creators often assume that features that alter access, ownership, and control of information will sell themselves because the features obviously benefit the organization. However, they overlook how users are rewarded or punished for sharing or withholding information-information politics that is almost never formally documented. . $^{40+11}$ If the physician receives no reward for using a decision support or expert system, or is not penalized for failing to use the system, then that physician is unlikely to share his or her decision-making authority.

Challenges to the physician monopoly of health consulting and physician values are powerful forces that help to drive physician opposition to clinical information systems and standards, as several social science theories would predict. ${ }^{13.14}$ The automation of medical information challenges physician domination of health care, and allows outsiders to question the physician's knowledge. ${ }^{26}$ If, as many experts claim, only $10 \%$ to $20 \%$ of medicine is known with certainty, knowledgeable experts may well disagree. ${ }^{+2-43}$ Given this uncertainty, physicians have been socialized to guide their practice from personal knowledge and experience. ${ }^{+4+45} \mathrm{~A}$ clinical expert system that recommends treatment regimens contrary to the individual physician's personal experience would threaten the validity of the physician's core value system.

\section{Managed Care's Impact on Physician Opposition}

As discussed earlier, physicians have controlled most health care spending in the fee-for-service environment. A physician with an income of $\$ 200,000$ has generated an estimated annual revenue stream (often for a hospital) as high as $\$ 1$ million in this environment. ${ }^{46}$ Virtually no hospital administration has mandated that physicians use computers, fearing that physicians opposed to clinical information systems would take their patients elsewhere and disrupt that revenue stream.

This tacit threat began to lose its power as payors demanded greater control of health care spending through managed care, certification, or compliance programs. Physicians are being replaced as the final arbiters of health care spending because under pressure from patients, hospitals, and others, they may become less likely to order testing or therapy that is not covered by third-party payment. In some decisions, such as in the application of high cost therapies such as magnetic resonance imaging, physicians must obtain pre-authorization. As more patients shift from unrestricted fee-for-service reimbursement to managed care, physicians lose autonomy.

Neither health plans nor government has full authority to direct patient treatment. Physicians do. Managed care plans achieve control by setting care standards through protocols and by implementing drug formularies. ${ }^{2,47-48}$ Protocols reduce physician autonomy and routinize some aspects of care, which enables nonphysicians to deliver more care. ${ }^{21,49-50}$ By insisting on and enforcing standards, large payors have provided financial incentives for physicians to conform to standards, in effect "rewarding" physicians for changing their behavior. ${ }^{51}$ Information systems technology adds a powerful tool for delivering and monitoring standards. ${ }^{43,52-53}$ When physicians use a clinical information system to determine and record patient care interactively, the system provides the ideal medium for communicating protocols and rules ${ }^{48.54-55}$ The U.S. Agency for Health Care Policy and Research, recognizing this fact, plans to translate its guidelines into computer formats. ${ }^{43,56}$ In addition, the clinical information system provides the capability to record adherence to the protocol.

\section{Penetration of Information Systems in Ambulatory Practice. Settings}

To determine the extent to which physicians have adopted clinical information systems, 522 nonhospital-based specialty or multispecialty groups with five or more physicians were randomly selected from the American Medical Association (AMA) group practice census to receive a survey. The subspecialties family/general, internal medicine, pediatrics, obstetrics/gynecology, psychiatry, cardiology, and gastroenterology were included because they were most likely to contract with MCOs. The survey was to the group practice administrator. The survey methodology included a presurvey letter, the survey, and a postcard reminder. All nonresponders were sent a second mailing of the survey and, finally, were contacted by telephone and faxed a copy of the survey.

Survey findings from the 197 respondents are presented. Adoption of 34 medical software applications was measured. In keeping with Leavitt and Whisler's framework, the findings group applications by three stages: (1) transaction; (2) decision support; and (3) simulation of human thought or expert systems. ${ }^{23}$ However, systems are often packaged by vendors with features that cross the stages of the framework, particularly in the case of stand-alone department systems such as pharmacy and laboratory. For example, virtually all pharmacy systems today include drug/drug and drug/allergy interactions, and most laboratory systems can automatically order an additional test dependent upon the results of an initial screening; these functions could be considered simulation of human thought.

As shown in Table 1 (see page 38), most nonhospital-based specialty group practices have adopted stage one transaction systems. These systems are the meat and potatoes of information management, holding fields of data at the greatest level of detail at which the data can be defined. Transaction systems that record patient name, address, billing, and insurance information are widely used. Systems that record data specific to the clinical aspects of patient encounters (other than ancillary test results) but that are not related to billing are more sporadically used.

As information systems advance and develop, transaction systems feed the data they capture into higher-level system applications with decision support or expert capabilities, which apply statistical techniques to databases for probability analyses. A lack of patient-specific clinical data makes migration into the 
decision support stage impractical. For example, a large-scale transaction database that includes the patient's complete medical record increases the likelihood that a complete medication history is available. A comprehensive medication history allows stage two or three support systems to check for duplicate prescriptions, overdosing, overtreatment, and adverse effects from drug interactions. ${ }^{57}$ Without large-scale transaction systems as a base, stage two or three support systems would require the user to enter all the known medications for the patient before the clinician could receive feedback on interactions or therapeutic duplications.

Transaction data for pharmacy appeared in computerized databases early on, but traditionally these databases have been separate from medical databases in both inpatient and ambulatory settings. When a physician gives a patient a prescription during an office visit, the physician may make a note in the paper medical record or the office computer system. However, the prescriptions are typically filled at community pharmacies, where detailed pharmacy transaction data is recorded. These transaction databases belong to the drug store chain and are rarely integrated with medical transaction databases.

Some pharmacy benefit management companies (PBMs) have made strides in this integrative activity, but as a whole, the PBM industry has yet to become the "health information managers" once predicted. Inpatient pharmacy charges typically originate in departmental pharmacy systems. Summary billing data will be pulled into the billing process, which will include data on the diagnosis-related grouping and final primary diagnosis. However, for both inpatients and outpatients, the detailed pharmacy transaction database may be limited to information only on drugs prescribed, days supply, dosage, the physician, the pharmacy, and the insurance payment. The diagnosis is often absent because the data were not required for reimbursement. ${ }^{58}$

The survey indicated that $18 \%$ of physician group practices use pharmacy management systems, but only $4 \%$ had systems for concurrent review (see Table 2). Because few physician practices capture comprehensive medication histories, the pharmacist is more likely to have an information system that provides useful feedback on drug interactions, duplications, and other medication-related information. Unfortunately, the authority to change the prescription rests with the physician, who does not receive this feedback. Changing the prescription requires additional communication between the pharmacist and the physician.

The last stage of the system adoption framework involves using systems that simulate human thinking-those with "expert" capabilities. As transaction databases feed decision support systems, those support systems set the stage for providing "expert" feedback. This next step involves providing information in a more specific patient context and making complex information more useful.

Broad consensus holds that the human mind can balance only a limited number of variables-possibly fewer than sevenbefore the amount of information makes it difficult to arrive at a consistent diagnosis and treatment decision. ${ }^{42.59}$ Computers do not have this limitation, and thus can be very helpful in evaluating an unlimited number of variables. For example, LDS Hospital

\begin{tabular}{|c|c|c|}
\hline TABLE 1 & $\begin{array}{l}\text { on Systems in Use by Nonhospital-Based Specialty Group } \\
\text { of Five or More Physicians }(n=197)\end{array}$ & \\
\hline Application & Survey Description & $\%$ \\
\hline Registration & Patient or member registration and basic demographic data & $97 \%$ \\
\hline Billing & Automated patient or member billing & $96 \%$ \\
\hline Electronic commerce & Electronic commerce (e.g., claims for Blue Cross/Blue Shield or other third parties) & $86 \%$ \\
\hline Scheduling & Patient or procedure scheduling and tracking & $82 \%$ \\
\hline Office visil coding & Procedure, diagnosis, or office visil coding (for uniform billing, reporting, etc.) & $75 \%$ \\
\hline Laboratory & Data links to external systems or departments-laboratory & $55 \%$ \\
\hline Link with hospital & Office system link with hospital information system & $53 \%$ \\
\hline E-mail & E-mail (other physicians, patients, outside consultants, etc.) & $45 \%$ \\
\hline Link to literature & Computerized professional literature (books, journals, citation databases, etc.) & $35 \%$ \\
\hline Authorization tracking & Managed care referral authorization tracking & $30 \%$ \\
\hline Radiology & Data links to external systems or departments-radiology & $26 \%$ \\
\hline Encounter (clerk transcribed) & Clerk transcribed physician office or telephone encounter (back-entered) & $23 \%$ \\
\hline Order entry & Order entry (e.g., lab, radiology, EKG, eic.), consults, and treatments & $20 \%$ \\
\hline Transcribed dictation & Transcribed physician dictation (viewable from outside the word processing system) & $16 \%$ \\
\hline Progress notes & Physician progress notes & $15 \%$ \\
\hline Encounter (other providers) & Data from other provider encounters (emergency room, home, hospital, etc.) & $14 \%$ \\
\hline Problem list & Master patient problem list & $12 \%$ \\
\hline History \& exam & Physician history, physical exam, and other findings & $11 \%$ \\
\hline Encounter (nonphysician) & Nonphysician licensed caregiver entered office or telephone encouncer (real time) & $8 \%$ \\
\hline Patient teaching & Patient teaching support (instruction and charting of instruction) & $6 \%$ \\
\hline Patient flowsheet & Patient flowsheet (diabetic, blood pressure, or other special purpose) & $6 \%$ \\
\hline Patient history questionnaire & Patient history questionnaire responses & $4 \%$ \\
\hline
\end{tabular}




\begin{tabular}{l|l|c}
\hline \multicolumn{1}{|c|}{ TABLE 2 } & \multicolumn{2}{c}{$\begin{array}{l}\text { Decision Support Systems in Use by Nonhospital-Based } \\
\text { Specialty Group Practices of Five or More Physicians ( } \mathrm{n=197)}\end{array}$} \\
\hline Application & Survey Description & $\%$ \\
\hline Pharmacy management & Pharmacy management (medication lists, drug interactions, print patient prescriptions) & $18 \%$ \\
Satisfaction tracking & Patient or member customer satisfaction tracking & $10 \%$ \\
Management audits \& queries & Medical management audits or research queries & $8 \%$ \\
Concurrent review & Concurrent review (e.g., quality indicators or case management) & $4 \%$ \\
\hline
\end{tabular}

\begin{tabular}{l|l|c}
\hline \multicolumn{1}{|c|}{ TABLE 3 } & $\begin{array}{c}\text { "Expert" Systems in Use by Nonhospital-Based Specialty Group } \\
\text { Practices of Five or More Physicians (n=197) }\end{array}$ & $\%$ \\
\hline Application & Survey Description & $19 \%$ \\
\hline Prevention reminders & Health prevention and screening reminders (e.g., mammography) & $1.1 \%$ \\
Telephone triage & Patient telephone triage system (e.g., "ask-a-nurse") & $5 \%$ \\
Protocols (administrative) & Administrative practice protocol/guidelines (for authorizing specialist or other services) & $3 \%$ \\
Protocols (interactive expert) & Interactive clinical protocol/guideline or "expert" system (automatically checks patient data) & $3 \%$ \\
Voice recognition dictation & Voice recognition dictation (transcribed automatically) & $3 \%$ \\
Protocols (stand-alone expert) & Stand-alone protocol/guideline or "expert" system (user enters patient data) & $3 \%$ \\
Protocols (drug) & Pharmacy drug protocols & $3 \%$ \\
\hline
\end{tabular}

in Utah reported that its Adult Respiratory Distress Syndrome ventilator management protocol, which manages 246 variables, increased patient survival rates from $9.5 \%$ to $44 \%$ and is being adopted in other hospitals. ${ }^{22,60.61}$

Table 3 demonstrates that the penetration of "expert" systems is minimal. Health prevention reminders, in use at $19 \%$ of the practices, had the highest level of penetration. Telephone triage systems ranked second (11\%), but the primary users of telephone triage systems are nurses. ${ }^{62.63}$ Computerized protocols have been adopted in no more than $3 \%$ to $5 \%$ of the group practices.

\section{Direct "Hands-On" Use of Information Systems By Physicians}

Survey respondents in each group practice were also asked to estimate actual "hands-on" use of computer systems by physicians. The average usage across survey respondents is presented in Table 4 (see page 40). In these group practices, the majority of physicians do not use a clinical information system to view results or other patient data. Use of systems by physicians to enter data or for expert advice is considerably lower (6\% to $8 \%$ ).

\section{Discussion}

The penetration of sophisticated information systems capable of interactive delivery of drug protocols is quite low (3\%). In addition, physician "hands-on" use of information systems for even viewing data is low. Interaction with a drug protocol in real time-the ideal circumstance to optimize drug orders and prescriptions-requires that physicians enter orders rather than simply view patient results, medical literacure, or drug formularies. That is the only way the physician can get immediate feedback on ways to optimize drug choices for the best outcomes at the lowest cost. Across group practices, physician use of information systems for entry or interaction with protocols is minimal
( $6 \%$ to $8 \%$ ). With such low levels of "hands-on" physician use, designing computer-based interactive interventions to improve utilization of the pharmacy drug benefit is analogous to sending electronic messages to recipients who never check their e-mail.

\section{Limitations}

The study has several limitations. Using an informant survey method can introduce error. The survey was addressed to the practice administrator as spokesperson for the practice. This person may not have understood which computer applications were used by the practice and/or may have not known the extent of physician use of computer applications.

In addition, the response rate for this survey was $38 \%$, which may have introduced a nonresponse bias. However, in largescale surveys, even a $50 \%$ response rate has been difficult to achieve, and the response rates seen in mailed surveys to group practices have been even lower. The AMA has had only a $35 \%$ response rate to its own annual group practice survey. Nonresponse bias presents a threat to validity and may limit external generalizability.

\section{Implications For Health Care Leadership}

Today, computerized drug protocols exist that are updated in real time based on laboratory results on drug resistance. ${ }^{66.67}$ However, if the system requires interactive use by physicians to provide the benefit, then caution is in order. From the physicians' point of view, computerized data sets could become the basis for regulation and monitoring of their practices. Depending on the environment, PBMs might want to consider a staged strategy that starts with paper-based drug protocols, preprinted prescription sheets, and online drug information before building to interactive computerized drug protocols. Financial incentives for use of drug protocols is another potentially effective 


\begin{tabular}{|l|c}
\hline \multicolumn{1}{|c|}{ TABLE 4. } & $\begin{array}{l}\text { Average Percent Physician Use of } \\
\text { Computers in Nonhospital-Based } \\
\text { Specialty Group Practices of Five or } \\
\text { More Physicians (n=197) }\end{array}$ \\
\hline Type of Use & Percent Physicians Within Group \\
\hline Viewing & $28 \%$ \\
\hline Management or research & $14 \%$ \\
\hline Entry & $8 \%$ \\
\hline Expert advice & $6 \%$ \\
\hline
\end{tabular}

strategy. For example, one study found financial incentives (denial of payment) reduced the use of injectable antibiotics by $60 \% .{ }^{63}$

Nonhospital-based physicians have not widely accepted information systems technology. As long as physicians continue to resist such systems, the benefit this technology could bring to health care will be largely unrealized, not only in the day-to-day delivery of cost-effective care, but also in the availability of health care information for analysis.

Little doubt exists that such large data sets present untapped information sources. For pharmacy, with $95 \%$ of drugstores computerized by the early 1990 s, very rich transaction data bases exist, with enormous potential for data mining ${ }^{69}$ The only information superior to this type of evidence-based knowledge comes through randomized controlled trials. ${ }^{3,70}$ The cost and time to retrieve and summarize this detailed clinical patient data from paper medical records is prohibitive; the cost of gleaning research data from manual primary sources has been estimated at approximately $\$ 65$ per record in 1982 dollars. ${ }^{71}$ Acquisition of this information is cost effective only if the information is readily available as a by-product of delivering care.

The implementation of information systems has failed at a rate of $50 \%$ or more. ${ }^{72.74}$ Nevertheless, health care organizations continue to invest millions of dollars in their development and support. Health care organizations surveyed by Modern Healthcare projected spending an average of $\$ 8$ million per year on information systems; over half the respondents projected spending more than $\$ 11$ million per year. ${ }^{75}$ Capturing, storing, and processing information accounts for an estimated one-third of all health care expenditures in the United States. ${ }^{76}$ Spending is high because the potential benefit is equally high. Data collection systems to automate the clinical record alone could save an estimated $\$ 40$ billion to $\$ 80$ billion annually. ${ }^{74}$

Clinical information systems must be adopted by the entire health care organization or by none of it. Maintaining dual systems to accommodate those who refuse to use the computerized record can increase costs by $130 \%$ to $240 \% .^{77}$ The estimated cost of supporting an electronic medical record is about $\$ 5,000$ per physician.$^{78}$ Although limited pilot projects can be supported on an interim basis, the organization ultimately must make a "go" or "no go" decision.

Fee-for-service plans are in decline; only 15\% of all active employees enrolled in indemnity plans for $1997 .{ }^{79}$ But where fee- for-service plans remain dominant, physicians retain significant power in deciding whether to implement clinical information systems. PBMs should initially select information management projects that do not require direct physician use. For example, beginning with computer applications that monitor filled prescriptions and provide retrospective feedback on costs to physicians might be preferable to implementing interactive drug protocols that would require physician use. Further implementations must be carefully planned to allow physicians time to build a level of comfort with and trust of the information system. Many of the benefits of information systems will be postponed until physicians become active users of such technology.

\section{REFERENCES}

1. Grady ML, Schwartz HA. Automated data sources for ambulatory care effectiveness research. Washington, DC: Department of Health \& Human Services 1993.

2. Miller RH, Luft HS. Estimating health expenditure growth under managed competition: Science, simulation, and scenarios. JAMA: 1995; 273: 656-63.

3. Pryor DB, Califf RM, Harrell FE, et al. Clinical data bases: Accomplishments and unrealized potential. Medical Care 1985; 23(5): 623-47.

4. Eisenberg JM. Doctors' decisions and the cost of medical care. Ann Arbor, MI: Health Administration Press, 1986.

5. Mechanic D. Sources of countervailing power in medicine. J Health Politics, Policy and Law 1991; 164: 85-98.

6. Salmon JW. Medical education. J Health Politics, Policy and Law 1986; l1(3): $54 \mathrm{l}-45$

7. Gross R. Intuition and technology as bases of medical decision-making. In Delkeskamp-Hayes C, Cutter MAG, eds. Science, technology, and the art of medicine. Norwell, MA: Kluwer Academic; 1993; 183-97.

8. Eddy DM, Billings J. The quality of medical evidence: Implications for quality of care. Health Affairs 1988; 7(1): 19-32.

9. Salmon JW, Dedhiya SD. The vital role of pharmacy benefit management in health services research. J Managed Care Pharm 1998; 4(1): 1-5

10. Anderson JG. Clearing the way ior physicians' use of clinical information systems. Communications of the ACM 1997; 40(8): 83-90.

11. Dick RS, Steen EB. The computer based patient record. Washington, DC: National Academy Press; 1991.

12. Sittig DF, Stead WW. Computer-based physician order entry: The state of the art. JAMA 1994; 1(2): 108-23.

13. FitzHenry F. Systems, protocols, and power |dissertation]. Chicago, IL: University of Illinois at Chicago, 1997.

14. FitzHenry F, Reichelt PA, Salmon JW. Knowledge technology and power exchanges among dominant and economic forces in health care (unpublished).

15. Davenport TH, Jarvenpaa S, Beers MC. Improving knowledge work processes. Sloan Management Review 1996; 53-65.

16. Gilbert S: In medicine of the 90's, house calls are to nurses, on the telephone. New York Times. 1995; B7.

17. Townes PG, Park B, Joyce J, et al. Implementation of electronic medical records: The "people" factor. J Amb Care Management 1992; 15(3): 30-43. 18. Anderson JG, Jay SJ, Schweer HM, et al. Why doctors don't use computers: Some empirical findings. In Anderson JG, eds. Use and impact of computers in clinical medicine. New York: Springer Verlag; 1987; 97-109.

19. Chessare JB, Tórok KE. Implementation of COSTAR in an academic group practice of general pediatrics. MD Computing 1993; 10(1): 23-27.

20. Kaplan B. The medical computing lag: Perceptions of barriers to the application of computers in medicine. In J Tech Assessment in Health Care 1987; 3(1): 123-36.

21.. Fischer PJ, Stratmann WC, Lundsgaarde HP, et al. User reaction to PROMIS: Issues related to acceptability of medical innovations. Anderson JG, editors. Use and impact of computers in clinical medicine. New York: Springer Verlag; 1987; 284-301.

22. Kaplan B. Development and acceptance of medical information systems 
An historical overview. J Health \& Hum Res Admin 1988; 11(1): 9-29. 23. Leavitt HJ, Whisler TL. Management in the 1980s. Harvard Business Review $1958 ; 36: 41 .-48$.

24. Markus ML, Robey D. Information technology and organizational change: Causal structure in theory and research. Management Science 1988; 345: 83-98. 25. American Hospital Association. American Hospital Association hospital staistics. 1997-1998 ed. Chicago, 1L: Healthcare InfoSource, Inc., 1998. 26. Morison I. The second curve. New York: Ballantine Books, 1996. 27. Brodman K, Van Woerkom AJ, Erdmann AJ, Goldstein LS. Interpretation of symptoms with a data-processing machine. Arch Intern Med 1959; 103: 116-22. 28. Miller PL, Artificial intelligence in medicine: An emerging discipline. Viller PL, eds. Selected topics in medical artificial intelligence. New York: Springer-Verlag, 1988; $1-9$.

29. Weaver RR. Computers \& medical knowledge. Boulder, CO: Westview Press, 1991.

30. Forsythe DE. Blaming the user in medical informatics: The cultural nature of scientific practice. Knowledge \& Sociely 1992; 995-111.

31. Teach RL, Shorliffe EH. An analysis of physician attitudes regarding computer-based clinical consultation systems. Computers \& Biomedical Research 1981; 14: 542-58

32. Singer J, Sacks HS, Lucente F, Chalmers TC. Physician attitudes toward applications of computer database systems. J Amer Med Asso 1983; 249 :

$1610-14$

33. In Computerization and the transformation of work. Dunlop C, Kling R.

Dunlop C, Kling R, eds. Computerization and controversy: Value conflicis and social choices. Boston: Academic Press; 1991: 182-99.

34. Gotlieb CC, Borodin A. Social issues in computing. London: Academic Press, 1973.

35. Markus ML. Systems in organizations. Boston: Pitman, 1984

36. Markus ML, Keil M. If we build it, they will come: Designing information systems that people want to use. Sloan Management Review 1994; 35(4): $11-25$ 37. Rind DM, Safran C. Real and imagined barriers to an electronic medical record. Proceedings, Annual Symposium on Computer Applications in Medical Care 1993; 1674-78

38. Anderson JG. Computerized medical record systems in ambulatory care. J Amb Care Management 1992; 15(3): 67-75.

39. Payne TH, Murphy G, Wagner EH. The ambulatory medical record project at Group Health Cooperative: What did a decade of experience teach us? J Amb Care Management 1992; 15(3): 44-54.

40. Strassmann PA. The squandered computer. New Canaan, CT: Information Economics Press, 1997.

41. Schrage $M$. The real problem with computers. Harvard Business Review $1997 ; 178-88$

42. James B. SCAMC Plenary Session I: Computers, data, and continuous quality improvement. New Orleans, LA: Seminar sponsored by the American Medical Informatics Association, 1995.

43. Millenson ML. Demanding medical excellence. Chicago: University of Chicago Press, 1997.

44. White KL. Frontiers in primary care: Healing the schism: Epidemiology, medicine, and the public's health. New York: Springer-Verlag, 1991.

45. Eddy DM. Balancing cost and quality in fee-for-service versus managed care. Health Affairs 1997; 16(3): 162-73.

46. Hafferty FW, McKinlay JB. The changing medical profession: An international perspective. New York: Oxford University Press; 1993: 210-26.

47. Kerr EA, Mittman BS, Hays RD, et al. Managed care and capitation in California-how do physicians at financial risk control their own utilization? Annals Int Med 1995; 123(7): 500-4.

48. Strickland D. The future of guidelines: Health reform, managed care, and computers will drive the use of guidelines in the years ahead. Business \& Health $1994 ; 1227-31$.

49. Ritzer $G$, Walczak D. Rationalization and deprofessionalization of physicians. Social Forces 1988; 67(1): 1-22.

50. White WD, Salmon JW, Feinglass J, eds. The changing doctor-patient relationship and performance monitoring: An agency perspective. The corporate transformation of health care: Perspectives and implications. Amityville: Baywood; 1994: 1-32.

51. Himmelstein DU, Woolhandler S. Extreme risk-the new corporate proposition for physicians. N Engl J Med 1995; 333: 1706-8.

52. Ellwood P, Lundberg GD. Managed care: A work in progress. J Amer Med Asso 1996 ; 276(13): 1083-86.

53. Given CW, Sprafka RJ. Screening the ambulatory care performance of family practice residents using a health information system. J Med Systems 1983; 7(3): 285-94.

54. Austin SM, Balas EA, Mitchell JA, Ewigman BG. Effect of physician reminders on preventive clinical care: Meta-analysis of randomized clinical trials. Proceedings, Annual Symposium on Computer Applications in Medical Care 1994; 18: 121-25.

55. Frame PS, Zimmer JG, Werth PL, et al. Computer-based vs manual health maintenance tracking: A controlled trial. Arch Fam Medi 1994; 3: 581-88.

56. Hadorn DC, McCormick K, Diokno A. An annotated algorithm approach to clinical guideline development. J Ameri Med Asso 1992; 267(24): 3311-14. 57. Overhage JM, Tierney WM, McDonald CJ. Design and implementation of the Indianapolis network for patient care and research. Bull Med Library Assoc 1995; 83(1): 48-56.

58. Gandhi SK, Salmon JW, Kong SX, Zhao SZ. Administrative databases and outcomes assessment: An overview of issues and potential utility. J of Managed Care Pharm 1999; 5(3)

59. Simon HA. The science of the artificial Cambridge, MA: MIT Press; 1969 60. Borzo G. First clinical trial of computerized protocol under way. Ameri Medi News 1993; 36: 11-12.

61. East TD, Bohm SH, Wallace CJ, et al. A successful computerized protocol for clinical managment of pressure control inverse ratio ventilation in ARDS patients. Chest 1992; 101: 697-710.

62. American Academy of Ambulatory Care Nursing. Nursing in ambulatory care: The future is here. Washington, DC: American Nurses Publishing, 1997. 63., Henry SB, Borchelt D, Schreiner JG, Musen MA. A computer-based approach to quality improvement for telephone triage in a community AIDS clinic. Nursing Admin Qly 1994; 18(2); 65-73.

64. Kaplan B. The influence of medical values and practices on medical computer applications. Anderson JG, ed. Use and impact of computers in clinical medicine. New York: Springer Verlag, 1987: 39-50.

65. Shuman TM. Hospital computerization and the politics of medical decision-making. Simpson RL, Simpson IH, ed. Research in the sociology of work: High tech work. Greenwich, CT: JAI Press; 1988: 261-87.

66. Evans RS, Pestotnik SL, Classen DC, Burke JP. Development of an automated antibiotic consultant. M.D.Computing 1993; 10(1): 1.7-22

67. Evans RS, Pestotnik SL, Classen DC, et al. A computer-assisted management program for antibiotics and other antiinfective agents. $N$ Engl J Med $1998 ; 338(4): 232-38$.

68. Brook RH, Williams $\mathrm{K}$. Effect of medical care review on the use of injections: A study of the New Mexico Experimental Care Review Organization. Annals of Int Med 1976; 85(4): 509-15.

69. Fayyad U, Piatetsky-Shapiro G, Smyth P. The KDD process for extracting useful knowledge from volumes of data. Communications of the ACM 1996 39: $27-34$

70. Ellwood P. A technology of patient experience. N Engl J Med 1988; 318 1549-56.

71. Institute of Medicine. Assessing medical technologies. Washington, DC: National Academy Press, 1985.

72. Friedman RB, Gustafson DH. Computers in clinićal medicine: A critical review. Computers and Biomed Res 1977; 10: 199-204.

73. Lyytinen K, Hirschheim RA. Information systems failure-A classification of empirical literature. Oxford Surveys in Infor Tech 1987; 4: 257-309.

74. Rifkin G. New momentum for electronic patient records. New York Times 1993; $8 \mathrm{~F}$

75. Morrissey J. Full speed ahead. Modern Healthcare 1996; 26(10): 97-108

76. Evans PB, Wurster TS. Strategy and the new economics of information. Harvard Business Review 1997; 71-82.

77. Schoenbaum SC, Barnett GO. Automated ambulatory medical records systems: An orphan technology. In J Tech Assessment in Health Care 1992; 8: 598-609.

78. Morrissey J. VHA delivers messaging. Modern Healthcare 1998; 34 79. Stein M. Health benefits cost rise just $0.2 \%$ in 1997 . Compensation and Benefits Management $1998 ; 14(4) ; 48-53$. 